\title{
Transcription in mosquito hemocytes in response to pathogen exposure Julián F Hillyer
}

Address: Department of Biological Sciences and Institute for Global Health, Vanderbilt University, VU Station B 35-1634, Nashville, TN 37235-1634, USA. Email: julian.hillyer@vanderbilt.edu

Published: 5 June2009

Journal of Biology 2009, 8:5 I (doi: I0.1 I86/jbioll5I)

The electronic version of this article is the complete one and can be found online at http://jbiol.com/content/8/5/5।

(C) 2009 BioMed Central Ltd

\begin{abstract}
Mosquito hemocytes are blood cells that are fundamental for combating systemic infection. A study published in BMC Genomics shows that hemocyte gene transcription in response to immune challenge is pathogen-specific and reaffirms the primary role of these cells in immunity.
\end{abstract}

\section{Mosquitoes and microbes}

Throughout their lifetime, mosquitoes are in danger of acquiring deadly pathogens. During their egg, larval and pupal stages, mosquitoes live in aquatic environments that are often rife with bacteria. Culex pipiens, for instance, thrive in sewer systems. As adults, mosquitoes often lose their legs, creating openings by which pathogens can enter their body. Female mosquitoes also engage in the dangerous behavior of biting vertebrates and ingesting their blood. This is done to acquire the nutrients necessary for the production of large numbers of eggs, but it exposes mosquitoes to bloodborne pathogens, such as Plasmodium, filarial nematodes and arboviruses. Besides being deadly and debilitating to humans, these organisms are pathogenic to mosquitoes if acquired in high enough numbers.

So how does a mosquito respond to a microbial pathogen? When a foreign invader enters the body cavity of a mosquito it elicits a systemic immune response. Similarly to that of vertebrates, this immune response has both humoral and cellular components. However, the invertebrate response lacks the properties of somatic hypermutation and immune memory that are hallmarks of vertebrate adaptive immunity. The mosquito cellular immune response includes phagocytosis and encapsulation by hemocytes (blood cells). The humoral response includes the phenoloxidase cascade system of melanization (an enzymatic process in which melanin polymers cross-link with proteins, sequestering pathogens and closing wounds), inducible antimicrobial peptides, reactive oxygen and nitrogen intermediates, and pattern recognition molecules. As with vertebrates, the line between cellular and humoral immunity is blurred because many humoral components are produced by hemocytes. Because of their involvement in both cellular and humoral pathways, the circulating nature of these cells and their ability to respond rapidly to an infection, it is now clear that hemocytes are the first line of defense against microbes that enter the hemocoel (body cavity) of the mosquito [1].

Given their fundamental role in immunity, it is surprising that little is known about the biology of mosquito hemocytes. This is probably because they are few in number and are difficult to manipulate. Much of what we know comes from studies that have morphologically and functionally characterized hemocyte subpopulations and described their role in pathogen killing and sequestration $[2,3]$. Other studies have focused on the discovery and 
characterization of individual genes and proteins, enabling in-depth investigations of a limited number of targets that were initially identified because of homology to genes with known function in other organisms [4]. However, a considerable percentage of the mosquito genes that have been identified either bioinformatically or through expressed sequence tag (EST) projects are of unknown function. Because single-gene approaches are unlikely to focus on these unknowns (many of which may be crucial), wholegenome transcriptomic and proteomic analyses are needed to narrow the field.

Recent studies have begun to exploit mosquito genomic data to screen thousands of genes simultaneously for transcriptional changes after various treatments. Initial work on mosquito hemocytes has included the characterization of transcriptional changes in hemocytes from the mosquito Armigeres subalbatus following infection with the filarial nematode Brugia malayi and in hemocytes from the mosquito Aedes aegypti following infection with live bacteria $[5,6]$. Clearly, additional work is needed in other medically important vectors to identify genes that are regulated in response to infection.

\section{The transcriptomic profile of Anopheles gambiae hemocytes}

In a recent article published in BMC Genomics, Baton et al. [7] present the first genome-wide transcriptomic analysis of the circulating hemocytes of the malaria vector Anopheles gambiae following natural infection with the rodent malaria parasite Plasmodium berghei and after immune challenge with heat-killed Escherichia coli and Micrococcus luteus. A total of 4,047 genes were found to be transcribed in hemocytes, of which 279 were present in at least two-fold higher abundance in hemocytes than in the rest of the body whereas 266 were found in lower abundance. Of the enriched transcripts, only $54.5 \%$ have predicted functions, highlighting the gap in our knowledge of mosquito biology. Of the genes with predicted functions, all components of the immune response were represented, including pattern recognition molecules, antimicrobial peptides, serine proteases, serine protease inhibitors, signal transduction proteins, stress response proteins, melanization-related molecules, redox/oxidoreductive molecules, and cytoskeletal organization and rearrangement (phagocytosis) proteins. Immune challenge with Plasmodium or bacteria resulted in the differential regulation of 959 genes, of which immunity-related genes were overrepresented whereas replication/transcription/translation-related genes were underrepresented, further showing that immune function is the primary role of hemocytes (Figure 1). When compared with previous studies, the transcriptome of A. gambiae hemocytes is

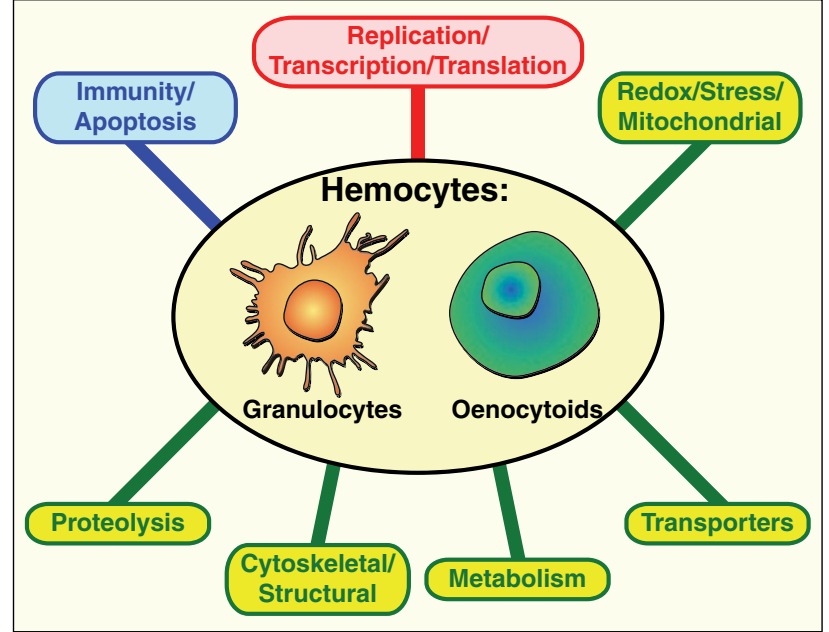

Figure I

Functional classification of genes transcribed in hemocytes. Among the genes transcriptionally regulated (up or down) following immune challenge, genes that function in immunity and apoptosis are overrepresented (blue) whereas genes that function in replication, transcription and translation are underrepresented (red). Genes in other functional classes (green) are not regulated at a higher or lower frequency than would be expected if there was no association between functional class and transcriptional regulation following challenge.

mostly consistent with the transcriptomic profile of other mosquito species but not with that of Drosophila $[6,8]$, illustrating evolutionary divergence within the order Diptera and underscoring the importance of directly studying insect species of vectorial significance.

\section{Differential immune response against pathogens}

Mosquitoes mount strong phagocytic immune responses against E. coli, whereas sequestration of M. luteus is primarily by melanization [3]. Plasmodium ookinetes (the motile zygotes of the parasite) in the midgut are killed by either lysis or melanization within 48 hours of infection [4]. In contrast, Plasmodium sporozoites (the infective stage) migrating through the hemocoel during the third week after infection are killed by mechanisms that have not been firmly characterized. However, the low levels of phagocytosis and melanization observed during migration suggest that most parasites are killed by some form of lytic mechanism [9].

These differences in the immune responses mounted against different pathogens are in agreement with the data presented by Baton et al. [7], which reveal distinct transcriptional signatures against two different bacterial species and between two stages of malaria parasites. After Plasmodium berghei infection, a total of 431 genes were differentially expressed in hemocytes. However, only $5.3 \%$ of the 
genes differentially expressed during Plasmodium infection were regulated in a similar manner for both the ookinete and sporozoite stages, whereas $3.7 \%$ of genes were regulated in opposite directions, indicating that more than $90 \%$ of genes were regulated exclusively during one of the two infection stages assayed. Genes involved in melanization were induced during ookinete invasion but not during sporozoite migration, consistent with previous reports that ookinetes often become melanized but that this rarely happens to sporozoites [4,9]. Interestingly, $37.2 \%$ of the immune genes regulated during sporozoite migration were members of the fibrinogen-related protein family (FREPs; also known as FBNs) of mosquitoes. This family is made up of 59 genes in A. gambiae, an expansion from the 14 genes found in Drosophila [10]. FBNs in Anopheles and other mosquitoes have been shown to be involved in antibacterial and anti-Plasmodium immunity, and it is tempting to speculate that their expansion was a consequence of continuous exposure to blood-borne pathogens.

After challenge with heat-killed E. coli or M. luteus, 641 transcripts were differentially regulated in hemocytes, but only $6.9 \%$ of those transcripts were similarly regulated in the two groups [7]. This was due mainly to a weaker response in transcriptional regulation following E. coli challenge, as $M$. luteus altered the transcriptional state of almost four times as many genes as E. coli. When only genes with putative immune function were analyzed, $7.7 \%$ of genes were differentially regulated in a similar manner. E. coli and $M$. luteus both induced genes involved in melanization, even though the latter pathogen was visually observed to elicit this immune process at a considerably higher rate. In addition, transcripts of genes involved in phagocytosis either decreased in abundance or were not regulated following immune challenge with $E$. coli, whereas transcription of several genes involved in this immune process increased in abundance after exposure to M. luteus, seemingly in conflict with the observation that phagocytic events are much more common against E. coli than M. luteus. It is probable that this is the result of different molecular interactions during the internalization of the two pathogens, including the possible requirement of melanization of $M$. luteus before the onset of phagocytosis [3].

Overall, the data presented by Baton et al. [7] are mostly consistent with previous transcriptomic analyses of the hemocytes of other mosquito species $[5,6]$, with the exception of the level of immune induction in A. gambiae hemocytes following challenge with heat-killed E. coli. Possible reasons for these discrepancies include mosquito species-specific differences or that inoculation with dead bacteria elicits a weaker response than infection with living bacteria. Furthermore, given that the rodent malaria parasite
Plasmodium berghei and the human malaria parasite Plasmodium falciparum elicit different midgut and carcass transcriptional profiles in response to ookinete invasion [11], future studies will need to address whether the hemocyte response now being reported [7] is similar to the response that occurs during infection with human malaria parasites. Nevertheless, the data presented by Baton et al. [7] provide a comprehensive dataset that will serve as a starting point for the functional characterization of numerous mosquito genes. The report of the breadth of genes transcribed by hemocytes, together with data on their cellular biology, supports the hypothesis that they form the primary component of the mosquito immune response [1-3,7].

\section{Application in transmission control strategies}

Plasmodium parasites, the causative agents of malaria, kill over a million people per year, and another 500 million people suffer from clinical disease. Currently, the control of mosquito-borne diseases has consisted of treating infected individuals, killing the mosquito vector and limiting vectorhuman contact. Although these approaches have reduced disease prevalence, their efficacy is diminishing, mainly because of the emergence of drug resistance by Plasmodium parasites and insecticide resistance in the insect vector. Thus, because of the reduced efficacy of current control methods, compounded by the failure to discover new drugs, insecticide replacements and effective vaccines, it has become necessary to develop new control strategies.

One possible strategy that has gained support in recent years is to genetically manipulate insect pests such that they are unable to transmit disease-causing pathogens, and to mass release them into the environment to displace natural populations of susceptible mosquitoes. Before such a strategy can be implemented several hurdles must be overcome, one of which is the identification of candidate mosquito genes that confer resistance to infection. The best candidate genes are probably transcribed in hemocytes, because these cells are involved in immune responses throughout the insect and even produce proteins with antiparasitic activity in the midgut [4]. The study by Baton et al. [7] provides a comprehensive dataset of gene transcription following Plasmodium infection and sets the stage for indepth functional studies on the role of candidate genes in fighting infection.

\section{Acknowledgements}

The author is funded by NSF grant IOS-08I 7644 .

\section{References}

I. Hillyer JF, Schmidt SL, Fuchs JF, Boyle JP, Christensen BM: Ageassociated mortality in immune challenged mosquitoes (Aedes 
aegypti) correlates with a decrease in haemocyte numbers. Cell Microbiol 2005, 7:39-5I.

2. Castillo JC, Robertson AE, Strand MR: Characterization of hemocytes from the mosquitoes Anopheles gambiae and Aedes aegypti. Insect Biochem Mol Biol 2006, 36:891-903.

3. Hillyer JF, Schmidt SL, Christensen BM: Hemocyte-mediated phagocytosis and melanization in the mosquito Armigeres subalbatus following immune challenge by bacteria. Cell Tissue Res 2003, 313:117-127.

4. Blandin SA, Marois E, Levashina EA: Antimalarial responses in Anopheles gambiae: from a complement-like protein to a complement-like pathway. Cell Host Microbe 2008, 3:364-374.

5. Aliota MT, Fuchs JF, Mayhew GF, Chen CC, Christensen BM: Mosquito transcriptome changes and filarial worm resistance in Armigeres subalbatus. BMC Genomics 2007, 8:463.

6. Bartholomay LC, Mayhew GF, Fuchs JF, Rocheleau TA, Erickson SM, Aliota MT, Christensen BM: Profiling infection responses in the haemocytes of the mosquito, Aedes aegypti. Insect Mol Biol $2007,16: 761-776$
7. Baton LA, Robertson A, Warr E, Strand MR, Dimopoulos G: Genome-wide transcriptomic profiling of Anopheles gambiae hemocytes reveals pathogen-specific signatures upon bacterial challenge and Plasmodium berghei infection. BMC Genomics 2009, 10:257.

8. Irving P, Ubeda JM, Doucet D, Troxler L, Lagueux M, Zachary D, Hoffmann JA, Hetru C, Meister M: New insights into Drosophila larval haemocyte functions through genome-wide analysis. Cell Microbiol 2005, 7:335-350.

9. Hillyer JF, Barreau C, Vernick KD: Efficiency of salivary gland invasion by malaria sporozoites is controlled by rapid sporozoite destruction in the mosquito haemocoel. Int J Parasitol 2007, 37:673-681.

10. Dong Y, Dimopoulos G: Anopheles fibrinogen-related proteins provide expanded pattern recognition capacity against bacteria and malaria parasites. J Biol Chem 2009, 284:9835-9844.

II. Dong Y, Aguilar R, Xi Z, Warr E, Mongin E, Dimopoulos G: Anopheles gambiae immune responses to human and rodent Plasmodium parasite species. PLoS Pathog 2006, 2:e52. 